EPJ Web of Conferences 108, 02028 (2016)

DOI: $10.1051 /$ epjconf/201610802028

(C) Owned by the authors, published by EDP Sciences, 2016

\title{
Quasiclassical Study of the Quantum Mechanical Two-Coulomb-Centre Problem
}

\author{
M. Hnatič ${ }^{1,2,3, a}$, V.M. Khmara ${ }^{3, b}$, V.Yu. Lazur ${ }^{4, c}$, and O.K. Reity ${ }^{4, d}$ \\ ${ }^{1}$ Department of Theoretical Physics, SAS, Institute of Experimental Physics, Watsonova 47, 04001 Košice, \\ Slovakia \\ ${ }^{2}$ Bogoliubov Laboratory of Theoretical Physics, Joint Institute for Nuclear Research, Joliot-Curie 6, 141980 \\ Dubna, Moscow Region, Russia \\ ${ }^{3}$ Faculty of Science, P. J. Šafárik University, Šrobárova 2, 04154 Košice, Slovakia \\ ${ }^{4}$ Department of Theoretical Physics, Uzhhorod National University, Voloshyna 54, 88000 Uzhhorod, Ukraine
}

\begin{abstract}
A recurrent scheme for finding the quasiclassical solutions of the onedimensional equation got by the separation of variables from the Schrödinger equation in prolate spheroidal coordinates has been elaborated. By means of this scheme, WKB expansion quasiclassical two-Coulomb-centre wave functions at large distances between the fixed positive charges (nuclei) have been constructed for the entire space of the negative particle (electron). Our method provides simple uniform estimates of the eigenfunctions at arbitrary internuclear distances $R, R \gg 1$ included. In contrast to the perturbation theory, the interaction need not be very small in the quasiclassical approximation. Its applicability domain is hence wider. That permits analyzing qualitative laws for the behavior and properties of quantum mechanical systems.
\end{abstract}

\section{Introduction}

The study of the electron motion in the field of two Coulomb centers (the so-called $Z_{1} e Z_{2}$ problem) began with the paper of Pauli [1]. Since then, this problem has attracted a lot of research mostly in connection with the problems of atomic and molecular physics (the status of the problem and references on the subject up to 1978 can be found in the monograph [2] and next papers [3, 4]).

The intensive studies of the problem of two Coulomb centers $Z_{1} e Z_{2}$ during the last thirty years were stimulated not only by the availability of powerful computers and the successes achieved with asymptotic methods in solving ordinary differential equations, but also by the requirements of mesomolecular physics [5, 6] and the theory of ion-atom collisions [7]. New forms of asymptotic solutions were obtained both for the problem of the hydrogen molecular ion $\mathrm{H}_{2}^{+}$[8] and for the problem of two centers with strongly different charges [9-11].

\footnotetext{
ae-mail: hnatic@saske.sk

be-mail: viktor.khmara@student.upjs.sk

ce-mail: volodymyr.lazur@uzhnu.edu.ua

de-mail: okreity@gmail.com
} 
The two-Coulomb-centre problem was also investigated in the relativistic case [12], the study of exchange interactions in molecular ion dimers [13], at small intercentre distances in two-dimensional [14] and arbitrary dimensional $[15,16]$ cases.

In spite of this, there is still a high interest in this problem for two main reasons. On the one hand, the two-Coulomb-centre system is an important model for the theory of diatomic molecules, much as the hydrogen atom is for the theory of multielectron atoms. On the other hand, this system has many applications, such as in the study of specific scattering problems and the characterization of the plasma radiation.

As a suitable method for calculating the wave functions and all other quantities required in the problem of the interaction of two heavy ions, we propose to employ the quasiclassical approach. This approach allows us to obtain analytic solutions, but it is limited to asymptotically large internuclear distances $R$. These distances should be sufficiently such large that the quantum penetrability of the potential barrier separating the atomic particles is much smaller than unity. A large number of problems exit [17-19], the solutions of which depend on that region of the internuclear distance. We stress, however, that the analytic expressions derived for the asymptotic behavior of various splittings and shifts of the potential curves can sometimes be used in the region of internuclear distances that are smaller than those given by the formal criteria of applicability of the asymptotic expansions. Qualitatively, this can be explained by the fact that the asymptotic solutions of the two-center problem retain the basic analytic properties of the exact solution [2] rather well, even the first term of the wave function expansion in powers of $R^{-1}$, up to sufficiently small $R$, and, thus, reproducing the results of variational calculations [19]. These properties are also conserved for other quantities computed with these functions.

\section{Basic equations}

The motion of the electron in the field of two fixed nuclei with charges $Z_{1}$ and $Z_{2}$ is described by the Schrödinger equation

$$
\left(-\frac{1}{2} \Delta-\frac{Z_{1}}{r_{1}}-\frac{Z_{2}}{r_{2}}\right) \Phi(\vec{r}, R)=E(R) \Phi(\vec{r}, R),
$$

where $r_{1}$ and $r_{2}$ are the distances from the electron to nuclei 1 and 2, $E(R)$ is the electron energy and $R$ is the distance between the nuclei. The Schrödinger equation (1) is separable in the prolate spheroidal coordinates:

$$
\begin{array}{ccc}
\xi=\left(r_{1}+r_{2}\right) / R, & \eta=\left(r_{1}-r_{2}\right) / R, \quad \phi=\arctan (y / x), \\
\xi \in[1 ; \infty), & \eta \in[-1 ; 1], \quad \phi \in[0 ; 2 \pi),
\end{array}
$$

where $x, y, z$ are the Cartesian coordinates of electron. If we replace the wave function $\Phi(\vec{r}, R)$ by the product function

$$
\Phi(\vec{r}, R)=\frac{U(\xi, R)}{\sqrt{\xi^{2}-1}} \frac{V(\eta, R)}{\sqrt{1-\eta^{2}}} \frac{e^{ \pm i m \phi}}{\sqrt{2 \pi}}=\frac{\Psi(\xi, \eta, R)}{\sqrt{\left(\xi^{2}-1\right)\left(1-\eta^{2}\right)}} \frac{e^{ \pm i m \phi}}{\sqrt{2 \pi}}
$$

and use the new variables

$$
\mu=\frac{R}{2}(\xi-1), \quad \mu \in[0, \infty), \quad v=\frac{R}{2}(1+\eta), \quad v \in[0, R],
$$


we obtain the quasiradial and quasiangular equations for functions $U(\xi, R)$ and $V(\eta, R)$

$$
\begin{gathered}
U^{\prime \prime}(\mu)-\left[\gamma^{2}-\frac{Z_{1}+Z_{2}+\lambda_{\xi} / R}{\mu}-\frac{Z_{1}+Z_{2}-\lambda_{\xi} / R}{R+\mu}+\frac{R^{2}\left(m^{2}-1\right)}{4 \mu^{2}(R+\mu)^{2}}\right] U(\mu)=0, \\
V^{\prime \prime}(v)-\left[\gamma^{2}-\frac{Z_{1}-Z_{2}-\lambda_{\eta} / R}{v}+\frac{Z_{1}-Z_{2}+\lambda_{\eta} / R}{R-v}+\frac{R^{2}\left(m^{2}-1\right)}{4 v^{2}(R-v)^{2}}\right] V(v)=0,
\end{gathered}
$$

where $\gamma=(-2 E)^{1 / 2}$.

These new functions satisfy the following boundary conditions:

$$
U(1)=0, \quad U(\xi) \underset{\xi \rightarrow \infty}{\longrightarrow} 0, \quad V( \pm 1)=0 .
$$

Here $\lambda_{\xi}$ and $\lambda_{\eta}$ are separation constants depending on $R$ and $m$ is the modulus of the magnetic quantum number. The two one-dimensional equations (5) and (6) are equivalent to the original Schrödinger equation provided the separation constants are equal:

$$
\lambda_{\xi}=\lambda_{\eta}
$$

When $R$ is much larger than the size of the electron shells centered on the left-hand nucleus, the ratios $\mu / R$ and $v / R$ are small quantities in the intra-atomic space. This allows us to use the perturbation theory to solve (5) and (6) in intra-atomic space and to find the separation constants $\lambda_{\xi}, \lambda_{\eta}$.

\section{Perturbation theory and the asymptotic behavior of two-Coulomb-centre quasiradial and quasiangular wave functions}

In this section we give the basic formulae for the asymptotic behavior of the two-Coulomb-centre quasiradial and quasiangular wave functions in the vicinity of the left-hand nucleus [20] which will be employed for sewing with the quasiclassical solutions of the $Z_{1} e Z_{2}$ problem obtained in the underthe-barrier region (see Section 4).

Let us assume that when $R$ tends to infinity, $\lambda$ has the same order as $R$. Then in the zero-order approximation (i.e. at $R=\infty$ ) the equation (5) takes the following form:

$$
u^{\prime \prime(0)}(\mu)-\left[\gamma^{2}-\frac{\varkappa_{1}}{\mu}+\frac{m^{2}-1}{4 \mu^{2}}\right] u^{(0)}(\mu)=0,
$$

where

$$
\varkappa_{1}=Z_{1}+Z_{2}+\lambda^{(0)} / R
$$

The solution of (8) satisfying the boundary condition set at $\mu \rightarrow 0$ is

$$
u^{(0)}(\mu)=N_{1}^{(0)} \exp (-\gamma \mu)(2 \gamma \mu)^{(m+1) / 2} F\left(\frac{m+1}{2}-\frac{\varkappa_{1}}{2 \gamma}, m+1,2 \gamma \mu\right),
$$

where $N_{1}^{(0)}$ is the normalization constant which is determined from the condition

$$
\int_{0}^{\infty}\left|u^{(0)}(\mu)\right|^{2} d \mu=1 \Rightarrow N_{1}^{(0)}=\left[\frac{\left(n_{1}+m\right) !}{n_{1} !(m !)^{2}\left(2 n_{1}+m+1\right)}\right]^{1 / 2}
$$


and $F(\alpha, \beta, x)$ is the confluent hypergeometric function. The solution (9) satisfies the boundary condition at infinity provided the parameter $(m+1) / 2-\varkappa_{1} / 2 \gamma$ equals to zero or a negative integer, $(m+1) / 2-\varkappa_{1} / 2 \gamma=-n_{1},\left(n_{1}=0,1,2, \ldots\right)$. Hence for the separation constant $\lambda^{(0)}(R)$ we obtain

$$
\lambda_{n_{1}}^{(0)}(R)=R\left[\gamma\left(2 n_{1}+m+1\right)-Z_{1}-Z_{2}\right] .
$$

To find the solution at large but finite values of the parameter $R$, following [20] we shall use the perturbation theory. In equation (5), we shall consider the energy as a parameter with a certain given value and the separation constant $\lambda$ as an eigenvalue of the corresponding operator. Then the computation of the corrections to the eigenvalue and eigenfunction can be done following the standard approach. We expand the desired wave function $U(\mu)$ into series with respect to the unperturbed wave functions $u_{n_{1}}^{(0)}(\mu)$,

$$
U(\mu)=\sum_{n_{1}^{\prime}} c_{n_{1}^{\prime}}(R) u_{n_{1}^{\prime}}^{(0)}(\mu)
$$

Substituting this expansion into (5), the multiplying by $u_{n_{1}}^{(0) *}$ and integrating, we find

$$
\begin{aligned}
& \left(\lambda-\lambda_{n_{1}^{\prime}}^{(0)}-\frac{1-m^{2}}{2}\right)\left\langle n_{1}^{\prime}\left|\mu^{-1}\right| n_{1}^{\prime}\right\rangle c_{n_{1}^{\prime}} \\
& =\sum_{k=0}^{\infty} \frac{(-1)^{k+1}}{R^{k}}\left[Z_{1}+Z_{2}-\lambda / R+(k+3) \frac{1-m^{2}}{4 R}\right] \sum_{n_{1}^{\prime \prime}}\left\langle n_{1}^{\prime}\left|\mu^{k}\right| n_{1}^{\prime \prime}\right\rangle c_{n_{1}^{\prime \prime}},
\end{aligned}
$$

where $\left\langle n_{1}^{\prime}\left|\mu^{-1}\right| n_{1}^{\prime}\right\rangle$ are the matrix elements of the operator $\mu^{k}$ defined by means of the unperturbed functions $u_{n_{1}}^{(0)}(\mu)$. Here the matrix elements of the operator $1 / \mu$ are diagonal. From (10) we may calculate any order of the corrections to the eigenvalue and eigenfunction (for details see [20]).

So within the perturbation theory one can find the asymptotic (for large $R$ ) solutions of the equations (5) and (6). Normalizing the total wave function $\Phi(\vec{r}, R)$ to unity we obtain the wave function of the electron moving near the first nucleus and perturbed by the second one up to $R^{-2}$ as

$$
\Psi^{\mathrm{pert}}(\mu, v)=C\left[f_{n_{1}}^{(0)}(\mu)+\sum_{p=1}^{2} \sum_{k=-p}^{p} c_{n_{1}+k}^{(p)} f_{n_{1}+k}^{(0)}(\mu)\right]\left[f_{n_{2}}^{(0)}(v)+\sum_{p=1}^{2} \sum_{k=-p}^{p} c_{n_{2}+k}^{(p)} f_{n_{2}+k}^{(0)}(v)\right],
$$

where

$$
f_{l}^{(0)}(x)=\left(\frac{(l+m) !}{l !(m !)^{2}(2 l+m+1)}\right)^{1 / 2}(2 \gamma x)^{(m+1) / 2} e^{-\gamma x} F(-l, m+1,2 \gamma x) .
$$

Here $n=n_{1}+n_{2}+m+1$ and for $p=1,2$ all of the $c_{n_{1,2}+k}^{(p)}$ coefficients have been found in [20]. The expression for the normalization constant $C$ is too cumbersome and not given here.

\section{WKB solutions of the quasiangular equation in inter-centre region}

We rewrite the quasiangular equation (6) as a one-dimensional Schrödinger-like equation:

$$
V^{\prime \prime}-\frac{q^{2}}{\hbar^{2}} V=0
$$

where $q=\sqrt{2\left(U_{\mathrm{eff}}-E\right)}$ and the function

$$
U_{\text {eff }}=-\frac{\tilde{Z}_{1}}{v}-\frac{\tilde{Z}_{2}}{R-v}+\frac{\hbar^{2}\left(m^{2}-1\right)}{8 v^{2}(1-v / R)^{2}}, \quad \tilde{Z}_{1,2}=\left[ \pm\left(Z_{1}-Z_{2}\right)-\lambda / R\right] / 2
$$


plays the role of the effective potential energy.

We seek the solution of (13) as:

$$
V=e^{S / \hbar} \sum_{k=0}^{\infty} \hbar^{k} \varphi^{(k)}
$$

Substituting (14) into (13) and equating to zero the coefficients of each power of the Planck constant $\hbar$, we arrive at a system for the unknown functions $S(v)$ and $\varphi^{(n)}(v)$

$$
\begin{aligned}
& \left(S^{\prime}\right)^{2}=q^{2}, \\
& 2 S^{\prime} \varphi^{(0)^{\prime}}+S^{\prime \prime} \varphi^{(0)}=0, \\
& 2 S^{\prime} \varphi^{(k+1)^{\prime}}+S^{\prime \prime} \varphi^{(k+1)}+\varphi^{(k)^{\prime \prime}}=0, \quad k=0,1,2, \ldots
\end{aligned}
$$

According to the general conditions of validity of the quasiclassical approximation [21], the potential barrier should be quite wide, i.e., $v_{2} \ll v_{3}$ where $v_{2,3}$ are the internal turning points which are found from the expansion in powers of $R^{-1}$ and solving the equation at $q=0$. This gives us the requirement

$$
\left[2\left(\tilde{Z}_{1}+\tilde{Z}_{2}\right)+\left(4 \tilde{Z}_{1}^{2}+\gamma^{2}\left(1-m^{2}\right)\right)^{1 / 2}+\left(4 \tilde{Z}_{2}^{2}+\gamma^{2}\left(1-m^{2}\right)\right)^{1 / 2}\right] /\left(2 \gamma^{2} R\right) \ll 1
$$

which can be always fulfilled at large $R$. Then one can sew the WKB solution (14) with the asymptotics found by expanding the perturbed function $\Psi^{\text {pert }}$ (11) by powers of $v^{-1}$ :

$$
\Psi(\mu, v) \underset{v_{2} \ll v \ll v_{m}}{\longrightarrow} \Psi^{\text {as }}(\mu, v) .
$$

Here $v_{m}$ is the maximum point of function $U_{e f f}$. In the under-the-barrier region $v_{2}<v<v_{3}$, the solution of (15) is the decreasing function

$$
S(v)=-\int_{v_{2}}^{v} q\left(v^{\prime}\right) d v^{\prime} .
$$

The linear equations (16) and (17) then yield

$$
\begin{aligned}
\varphi^{(0)} & =\frac{C^{(0)}}{\sqrt{q}}, \\
\varphi^{(k)} & =\frac{1}{\sqrt{q}}\left[\int \frac{\varphi^{(k-1)^{\prime \prime}}}{2 \sqrt{q}} d v+C^{(k)}\right] .
\end{aligned}
$$

Calculating the integrals in $\varphi^{(k)}$ and applying the boundary condition (19) we find that $\varphi^{(2)} \sim R^{-3}$. Therefore, restricting ourselves by terms of order $R^{-2}$ we obtain the quasiclassical wave function in the under-the-barrier region,

$$
V=\frac{C^{(0)}}{\sqrt{q}} \exp \left[-\int_{v_{2}}^{v} q d v\right]\left(1-\frac{\tilde{Z}_{1}}{4 \gamma^{3} v^{2}}+\frac{\tilde{Z}_{2}}{4 \gamma^{3}(R-v)^{2}}\right),
$$

where

$$
\begin{gathered}
C^{(0)}=C(R)(-1)^{n_{2}} e^{-\tilde{Z}_{1} / \gamma} Q_{+} Q_{-}\left[1+\frac{C_{1}}{2 \gamma R}+\frac{C_{2}}{8 \gamma^{2} R^{2}}\right], \\
Q_{ \pm}=\left(\frac{\tilde{Z}_{1}}{\gamma} \pm \frac{\sqrt{m^{2}-1}}{2}\right)^{\left\{n_{2}+1 /[1 \pm \sqrt{(m-1) /(m+1)}] / / 2\right.}, \quad C_{1}=-\left[2 n_{2}+m+1-2\left(Z_{1}-Z_{2}\right) / \gamma\right],
\end{gathered}
$$




$$
\begin{array}{r}
C_{2}=\frac{\left(Z_{1}-Z_{2}\right)^{2}}{\gamma^{2}}\left[2\left(2 n_{2}+m-1\right)+\frac{\left(m^{2}-1\right)\left(2 n_{2}+m+1\right)}{2 n_{2}\left(n_{2}+m+1\right)+m+1}\right]-\frac{2\left(Z_{1}-Z_{2}\right)}{\gamma}\left[2 n_{2}\left(2 n_{2}+2 m-1\right)\right. \\
\left.+m^{2}-m-3-2 \frac{6 n_{2}\left(n_{2}+m+1\right)+(m+1)(m+2)}{2 n_{2}+m+1}\right]+2 n_{2}\left(2 n_{2}^{2}-7 n_{2}-9\right)+2 m^{2}\left(n_{2}-1\right) \\
+m\left(6 n_{2}^{2}-14 n_{2}-9\right)-7 .
\end{array}
$$

\section{Conclusions}

The quasiradial and quasiangular wave functions are derived for the electron moving in the field of two Coulomb centres with arbitrary charges, $Z_{1}$ and $Z_{2}$, when the distance between the centres is large. We used the quasiclassical approach to find the quasiangular wave functions and the modified perturbation theory for the quasiradial ones. In the present study, the first three terms of this expansion have been derived.

\section{Acknowledgements}

This work is supported by Slovak VEGA grant 1/0222/13.

\section{References}

[1] W. Pauli, Ann. Phys. (Leipzig) 68, 177-240 (1922)

[2] I.V. Komarov, L.I. Ponomarev, and S.Yu. Slavyanov, Spheroidal and Coulomb Spheroidal Functions (Nauka, Moscow, 1976) [in Russian]

[3] L.I. Ponomarev, T.P. Puzynina, and L.N. Somov, J. Phys. B10, 1335 (1977)

[4] L.I. Ponomarev, T.P. Puzynina, and N.F. Truskova, J. Phys. B11, 3861 (1978)

[5] L.I. Men'shikov and L.N. Somov, Sov. Phys. Usp. 33, 616-646 (1990)

[6] S.S. Gershtein, Yu.V. Petrov, and L.I. Ponomarev, Sov. Phys. Usp. 33, 591-615 (1990)

[7] R.K. Janev, L.P. Presnyakov, and V.P. Shevelko, Physics of Highly Charged Ions (Springer, 1985)

[8] E.A. Solov'ev, Sov. Phys. Usp. 32, 228-250 (1989)

[9] I.V. Komarov and E.A. Solov'ev, Theor. Math. Phys. 40, 645-649 (1979)

[10] T.P. Grozdanov, R.K. Janev, and V.Yu. Lazur, Phys. Rev. A 32, 3425 (1985)

[11] V.Yu. Lazur, Yu.Yu. Mashika, R.K. Janev, and T.P. Grozdanov, Theor. Math. Phys. 87, 401-410 (1991)

[12] O.K. Reity, V.Yu. Lazur, and A.V. Katernoha, J. Phys. B 35, 1-17 (2002)

[13] M.J. Jamieson et al, J. Phys. B. 42, 095203 (2009)

[14] D.I. Bondar, M. Hnatič, and V.Yu. Lazur, Theor. Math. Phys. 148, 1100-1116 (2006)

[15] D.I. Bondar, M. Hnatič, and V.Yu. Lazur, J. Phys. A 40, 1791-1807 (2007)

[16] D.I. Bondar, M. Hnatič, and V.Yu Lazur, Phys. Part. Nucl. Lett. 5, 255-258 (2008)

[17] B.M. Smirnov, Asymptotic Methods in the Theory of Atomic Collisions (Atomizdat, Moscow, 1973) [in Russian]

[18] E.E. Nikitin and B.M. Smirnov, Slow Atomic Collisions (Energoatomizdat, Moscow, 1990) [in Russian]

[19] M.I. Chibisov and R.K. Janev, Phys. Rep. 166, 1-87 (1988)

[20] T.M. Kereselidze, Z.S. Machavariani, and I.L. Noselidze, J. Phys. B 29, 257-273 (1996)

[21] L.D. Landau and E.M. Lifshitz, Quantum Mechanics (Pergamon, Oxford, 1965) 